\title{
Efeitos de diferentes condições de umidade do solo e profundidades de germinação de Brachiaria plantaginea e Digitaria spp. sobre a eficácia do herbicida amicarbazone
}

\section{Effects of different soil humidity conditions and germination depths of Brachiaria plantaginea and Digitaria spp. over amicarbazone herbicide efficacy}

\author{
Caio Antonio Carbonari ${ }^{1}{ }^{*}$, Roberto Estevão Bragion de Toledo ${ }^{2}$, Edivaldo Domingues Velini $^{3}$, \\ Eduardo Negrisoli ${ }^{4}$, Marcelo Rocha Correa ${ }^{4}$, Caio Vitagliano Santi Rossi ${ }^{5}$
}

Resumo - Um experimento foi realizado em casa-de-vegetação, com o objetivo de avaliar a eficácia do herbicida amicarbazone no controle de Brachiaria plantaginea e Digitaria spp. em função da profundidade de germinação e condições de umidade do solo. Os tratamentos foram constituídos por cinco profundidades de germinação das plantas daninhas $(1 ; 2 ; 3 ; 4$ e $5 \mathrm{~cm}) \mathrm{e}$ quatro condições de umidade (15 mm de chuva seguido da aplicação de herbicida; $15 \mathrm{~mm}$ de chuva seguido de aplicação de herbicida e $15 \mathrm{~mm}$ de chuva aos 7 dias após a aplicação (DAA); $15 \mathrm{~mm}$ de chuva seguido de aplicação de herbicida e $15 \mathrm{~mm}$ de chuva aos 14 DAA; aplicação do produto em solo seco e $15 \mathrm{~mm}$ de chuva aos 14 DAA), e testemunha sem aplicação do herbicida, dispostos em esquema fatorial 5 x 5, com quatro repetições. As sementes das plantas daninhas foram semeadas em vasos nas diferentes profundidades de germinação e realizaram-se avaliações visuais de controle das plantas aos 14, 28 e 42 dias após a apliçação (DAA). Observou-se que para $B$. plantaginea, os melhores resultados de controle ocorreram nos tratamentos com a ocorrência de chuvas, sendo observadas falhas iniciais no controle, quando o produto foi aplicado em solo úmido e sem ocorrência de chuvas nas profundidades de 1,2 e $5 \mathrm{~cm}$, com a recuperação e bons níveis de controle aos final do estudo. Para Digitaria spp. foram observados bons níveis de eficácia de controle das plantas, nas diferentes condições de aplicação.

Palavras-chave: Planta daninha, lixiviação, adsorção.

\begin{abstract}
An experiment was conducted in green house, with the objective of evaluating the efficacy of amicarbazone herbicide in controlling Brachiaria plantaginea and Digitaria spp., in function of germination depth and soil humidity conditions. Treatments were constituted by five germination depths of weeds $(1 ; 2 ; 3 ; 4$ e $5 \mathrm{~cm}$ ) and four humidity conditions (15 mm of rainfall followed by herbicide application; $15 \mathrm{~mm}$ of rainfall, followed by herbicide application and 15 $\mathrm{mm}$ of rainfall after 7 days after application (DAA); $15 \mathrm{~mm}$ of rainfall, followed by herbicide application and $15 \mathrm{~mm}$ of rainfall at 14 days after application (DAA); herbicide application on dry soil and $15 \mathrm{~mm}$ of rainfall at 14 DAA, and check without herbicide application, disposed in

\footnotetext{
$1_{*}^{*}$ Eng. Agr. Dr., Depto. Produção Vegetal, FCA/Unesp, Fazenda Experimental Lageado, Caixa Postal 237, 18603970, Botucatu-SP, carbonari@fca.unesp.br

${ }^{2}$ Eng.-Agr. Dr., Piracicaba-SP.

${ }^{3}$ Depto. Produção Vegetal, FCA/Unesp, Fazenda Experimental Lageado, Caixa Postal 237, 18603-970, Botucatu-SP.

${ }^{4}$ Agr Dr. TechField-Nupam/FCA/Unesp, Fazenda Experimental Lageado, 18603-970, Botucatu-SP.

${ }^{5}$ Eng.Agr. Dr., Uberlândia-SP.
} 
factorial scheme $5 \times 5$, with four replications. Weeds seeds were seeded in vases in different depths of germination and visual evaluations of plants control were conducted at 14, 28 and 42 days after application (DAA). It was observed that for $B$. plantaginea, the best control results occurred in treatments with rains occurrence, being observed initials flaws in controlling when the product was applied in wet soil and without rains occurrence in 1,2 and $5 \mathrm{~cm}$ of depth, with recover and superior control levels at the end of the study. For Digitaria spp., satisfactory levels of efficacy on weeds control were observed in the different application conditions.

Key-words: weed, leaching, adsorption.

\section{Introdução}

Em sistemas agrícolas, o solo representa o destino final dos herbicidas aplicados diretamente a ele ou à parte aérea das plantas. No solo, os herbicidas podem sofrer adsorção, lixiviação, degradação através de processos físicos (volatilização e fotodecomposição), químicos e biológicos, além da absorção pelas plantas daninhas ou cultivadas. A compreensão de tais processos é fundamental para a previsão do comportamento dos herbicidas nos mais variados tipos de solo, para a seleção de doses adequadas e para se evitar efeitos prejudiciais dos herbicidas sobre culturas subsequentes (Velini, 1992).

Segundo Javaroni et al. (1999), a biodisponibilidade de herbicidas aplicados ao solo é essencialmente governada pelo equilíbrio dinâmico existente entre os processos de adsorção dos compostos presentes nos colóides do solo e sua solubilidade na fase solo-água. Como conseqüência, uma considerável influência desses parâmetros sobre a ação seletiva desses herbicidas deve ser esperada.

Em diversas situações podem ser observadas falhas no controle de diversas plantas daninhas em áreas tratadas com herbicidas residuais. Tais falhas podem estar relacionadas ao posicionamento do produto no perfil do solo, em função da ocorrência de chuvas. Carbonari et al. (2006) verificaram para Brachiaria decumbens que, a aplicação do amicarbazone em solo úmido seguido de período seco, sem ocorrência de chuvas, proporcionou um controle inicial de $93 \%$, $91 \%, 86 \%, 80 \%$ e $79 \%$, respectivamente, para as profundidades de $0-1,1-2,2-3,3-4$ e $4-5$ $\mathrm{cm}$. No entanto, após a ocorrência de chuvas, bons níveis de controle foram observados, independente da profundidade de germinação.

$\mathrm{O}$ amicarbazone apresenta solubilidade elevada em água (4600 ppm ou $\mathrm{mg} \mathrm{L}^{-1}$ à temperatura de $25^{\circ} \mathrm{C}$ e $\mathrm{pH}=4$ a 9), de baixa a moderada capacidade de adsorção no solo $(\mathrm{Koc}=23$ a 37), sendo classificado pelo IBAMA como herbicida de mobilidade alta na solo. Apresenta fotodegradação desprezível, e pressão de vapor de 0,975 x $10^{-}$ $8 \mathrm{~mm} \mathrm{Hg}\left(1,3 \times 10^{-6} \mathrm{~Pa}\right)$ à temperatura de $20^{\circ} \mathrm{C}$, o que o caracteriza como um herbicida praticamente não volátil e sua degradação ocorre primariamente através de dissipação, atribuída à degradação microbiana; sua meiavida varia de 3 a 6 meses, dependendo das condições de solo e clima, da dose, tipo e textura do solo, teor de matéria orgânica e quantidade de chuvas (TOLEDO et al., 2002; TOLEDO et al., 2004; ALMEIDA \& RODRIGUES, 2005).

O objetivo deste estudo foi avaliar a eficácia do herbicida amicarbazone no controle de Brachiaria plantaginea e Digitaria spp. em função da profundidade de germinação destas espécies, das condições de umidade do solo e períodos de seca. 


\section{Material e métodos}

Foi conduzido um experimento em casa-de-vegetação no NUPAM - Núcleo de Pesquisas Avançadas em Matologia da Faculdade de Ciências Agronômicas da UNESP, Campus de Botucatu/SP. Os experimentos foram realizados em vasos com capacidade para $2 \mathrm{~kg}$ de solo e dimensões de $15 \mathrm{x} 15 \mathrm{~cm}\left(0,0225 \mathrm{~m}^{2}\right)$ e $15 \mathrm{~cm}$ de profundidade. Os vasos foram preenchidos totalmente com solo do tipo Latossolo Vermelho Distrófico (LVd) de textura média, adubado mediante análise prévia de suas características. As características granulométricas do solo eram $20 \%$ de argila, $4 \%$ de silte e $76 \%$ de areia.

$\mathrm{O}$ herbicida amicarbazone (Dinamic) foi aplicado na dose de $1225 \mathrm{~g}$ i.a. ha ${ }^{-1}$ e, como tratamentos, foram avaliados os efeitos de cinco profundidades de germinação das plantas daninhas $(1 ; 2 ; 3 ; 4$ e $5 \mathrm{~cm})$ e aplicação do produto sob quatro condições de umidade do solo (15 mm de chuva, seguido da aplicação; $15 \mathrm{~mm}$ de chuva, seguido de aplicação e 15 $\mathrm{mm}$ de chuva 7 DAA; $15 \mathrm{~mm}$ de chuva, seguido de aplicação e $15 \mathrm{~mm}$ de chuva 14 DAA e aplicação do produto em solo seco e 15 mm de chuva 14 DAA), além de uma testemunha sem aplicação do herbicida, dispostos em um esquema fatorial $5 \times 5$, com quatro repetições.

As plantas daninhas utilizadas foram Brachiaria plantaginea e Digitaria spp. sendo semeadas nas diferentes profundidades em quantidades suficientes para uma germinação de 25 plantas por vaso, determinadas por teste prévio de germinação. As sementes foram posicionadas nas diferentes profundidades e posteriormente os vasos foram preenchidos com solo.

A aplicação do herbicida e simulação de chuva foram realizadas em um simulador estacionário de pulverização e precipitação localizado em laboratório fechado e com condições climáticas controladas. A manutenção da umidade dos vasos após simulação das chuvas foi realizada através de canos de PVC perfurados e posicionados no centro dos vasos, o que proporcionou $\mathrm{o}$ umedecimento sub-superficial dos vasos.

Foram realizadas avaliações aos 14, 28 e 42 dias após a aplicação (DAA), por meio de notas visuais de controle, baseadas em uma escala percentual, onde "0\%" representa nenhum controle e " $100 \%$ " o controle com morte de todas as plantas. Os resultados obtidos foram submetidos à análise de variância pelo teste $\mathrm{F}$ (5\% probabilidade) e as médias foram comparadas pelo teste Tukey (5\% probabilidade).

\section{Resultados e discussão}

Nas Tabelas 1, 2 e 3 estão apresentados os resultados de controle das plantas de $B$. plantaginea nas diferentes profundidades de germinação pelo herbicida amicarbazone aos 14, 28 e 42 DAA, respectivamente. Verifica-se na Tabela 1, aos 14 DAA, que os melhores resultados de controle nas diferentes profundidades foram observados quando ocorreu chuva aos 7 DAA e para aplicação em solo seco e irrigado sub-superficialmente. Observou-se também aos 14 DAA que, as maiores falhas no controle ocorreram aos $5 \mathrm{~cm}$ nos tratamentos aplicados em solo úmido e sem chuvas (até 14 DAA).

Aos 28 DAA (Tabela 2) os melhores resultados de controle foram observados nos tratamentos com a ocorrência de chuvas posterior à aplicação. As maiores falhas no controle foram observadas quando o produto foi aplicado em solo úmido e sem ocorrência de chuvas nas profundidades de 4 e $5 \mathrm{~cm}$. Na Tabela 3 (42 DAA), observa-se excelentes níveis de controle mesmo nas condições onde ocorreram falhas iniciais, demonstrando uma 
boa eficácia do produto no controle das plantas de B. plantaginea.

Tabela 1. Porcentagem de controle das plantas de Brachiaria plantaginea nas diferentes profundidades de germinação pelo herbicida amicarbazone, aos 14 DAA. Botucatu/SP 2006/07.

\begin{tabular}{|c|c|c|c|c|c|}
\hline \multirow{2}{*}{ Condições de Umidade } & \multicolumn{5}{|c|}{ Profundidade $(\mathrm{cm})$} \\
\hline & 1 & 2 & 3 & 4 & 5 \\
\hline chuva de 15 mm - aplicação & $77,5 \mathrm{~A} \mathrm{a}$ & $73,5 \mathrm{Ba}$ & $82,3 \mathrm{Aa}$ & $89,5 \mathrm{Aa}$ & $57,0 \mathrm{Bb}$ \\
\hline chuva (15 mm) - aplicação - chuva $(15 \mathrm{~mm}) 7^{1}$ & $88,2 \mathrm{~A} \mathrm{a}$ & $98,0 \mathrm{Aa}$ & $98,8 \mathrm{Aa}$ & $91,5 \mathrm{Aa}$ & $89,5 \mathrm{Aa}$ \\
\hline chuva (15 mm) - aplicação - chuva (15mm) $14^{1}$ & $73,0 \mathrm{Aab}$ & $88,0 \mathrm{Aa}$ & $86,3 \mathrm{Aa}$ & $61,3 \mathrm{Bb}$ & $66,3 \mathrm{Bb}$ \\
\hline aplicação solo seco - chuva $(15 \mathrm{~mm}) 14^{1}$ & $85,0 \mathrm{Aa}$ & $89,5 \mathrm{Aa}$ & $92,3 \mathrm{Aa}$ & $72,5 \mathrm{ABa}$ & $74,5 \mathrm{ABa}$ \\
\hline $\mathrm{F}$ (profundidade) & & & $3,38 *$ & & \\
\hline F (umidade) & & & $10,05 * *$ & & \\
\hline F (prof. x umidade) & & & $2,75^{*} *$ & & \\
\hline C.V. $(\%)$ & & & 17,47 & & \\
\hline D.M.S. & & & 19,85 & & \\
\hline
\end{tabular}

Médias seguidas de mesma letra maiúscula na coluna e minúscula na linha, não diferem estatisticamente entre si pelo teste Tukey $(\mathrm{P}<0,05)$ * - significativo a 5\% de probabilidade; ** - significativo a 10\% de probabilidade; ns não significativo. DAA: ${ }^{1}$ Dias após a aplicação.

Tais resultados também foram maiores falhas iniciais no controle de plantas observados a campo por Carbonari et al. de Brachiaria decumbens nas maiores (2006), onde a aplicação do herbicida profundidades de germinação $(3$ a $5 \mathrm{~cm}$ ), amicarbazone sobre solo úmido e seguido de atingindo, no entanto, bons níveis de controle longo período de estiagem, proporcionou após a ocorrência de chuvas.

Tabela 2. Porcentagem de controle das plantas de Brachiaria plantaginea nas diferentes profundidades de germinação pelo herbicida amicarbazone, aos 28 DAA. Botucatu/SP - 2006/07.

\begin{tabular}{|c|c|c|c|c|c|}
\hline \multirow[b]{2}{*}{ Condições de Umidade } & \multicolumn{5}{|c|}{ Profundidade $(\mathrm{cm})$} \\
\hline & 1 & 2 & 3 & 4 & 5 \\
\hline chuva de 15 mm - aplicação & $84,5 \mathrm{Aa}$ & $72,5 \mathrm{Bba}$ & $88,5 \mathrm{Aa}$ & $71,5 \mathrm{Bab}$ & $63,3 \mathrm{Bb}$ \\
\hline chuva (15 mm) - aplicação - chuva $(15 \mathrm{~mm}) 7^{1}$ & $99,5 \mathrm{Aa}$ & $98,3 \mathrm{Aa}$ & $99,8 \mathrm{Aa}$ & $94,3 \mathrm{Aa}$ & $89,75 \mathrm{Aa}$ \\
\hline chuva (15 mm) - aplicação - chuva $(15 \mathrm{~mm}) 14^{1}$ & $100,0 \mathrm{Aa}$ & $100,0 \mathrm{Aa}$ & $100,0 \mathrm{Aa}$ & $89,5 \mathrm{Aa}$ & $88,8 \mathrm{Aa}$ \\
\hline aplicação solo seco - chuva $(15 \mathrm{~mm}) 14^{1}$ & $99,5 \mathrm{Aa}$ & $99,8 \mathrm{Aa}$ & $95,5 \mathrm{Aa}$ & $86,3 \mathrm{ABa}$ & $96,5 \mathrm{Aa}$ \\
\hline F (profundidade) & & & $3,06 *$ & & \\
\hline F (umidade) & & & $12,11 * *$ & & \\
\hline F (prof. x umidade) & & & $0,54^{\mathrm{ns}}$ & & \\
\hline C.V. $(\%)$ & & & 13,99 & & \\
\hline D.M.S. & & & 17,97 & & \\
\hline
\end{tabular}

Nas Tabelas 4, 5 e 6 estão apresentados os resultados de controle das plantas de Digitaria spp., nas diferentes profundidades de germinação, pelo herbicida amicarbazone aos 14,28 e 42 DAA, respectivamente. Verifica-se nas Tabelas 4 e 5 , $(14$ e 28 DAA, respectivamente), excelentes níveis de eficácia de controle da planta daninha em todas as profundidades para as diferentes condições de aplicação. 
Tabela 3. Porcentagem de controle das plantas de Brachiaria plantaginea nas diferentes profundidades de germinação pelo herbicida amicarbazone, aos 42 DAA. Botucatu/SP 2006/07.

\begin{tabular}{|c|c|c|c|c|c|}
\hline \multirow[t]{2}{*}{ Condições de Umidade } & \multicolumn{5}{|c|}{ Profundidade $(\mathrm{cm})$} \\
\hline & 1 & 2 & 3 & 4 & 5 \\
\hline chuva de $15 \mathrm{~mm}$ - aplicação & 99,0 Aa & $100,0 \mathrm{Aa}$ & $100,0 \mathrm{Aa}$ & $98,3 \mathrm{Aa}$ & $98,8 \mathrm{Aa}$ \\
\hline chuva $(15 \mathrm{~mm})$ - aplicação - chuva $(15 \mathrm{~mm}) 7^{1}$ & $99,3 \mathrm{Aa}$ & $100,0 \mathrm{Aa}$ & $100,0 \mathrm{Aa}$ & $97,5 \mathrm{Ab}$ & $96,5 \mathrm{Aa}$ \\
\hline chuva $(15 \mathrm{~mm})$ - aplicação - chuva $(15 \mathrm{~mm}) 14^{1}$ & $100,0 \mathrm{Aa}$ & $100,0 \mathrm{Aa}$ & $100,0 \mathrm{Aa}$ & $98,5 \mathrm{Aa}$ & $99,0 \mathrm{Aa}$ \\
\hline aplicação solo seco - chuva $(15 \mathrm{~mm}) 14^{1}$ & $100,0 \mathrm{Aa}$ & $100,0 \mathrm{Aa}$ & $100,0 \mathrm{Aa}$ & $99,8 \mathrm{Aa}$ & $99,0 \mathrm{Aa}$ \\
\hline $\begin{array}{l}\text { F (profundidade) } \\
F \text { (umidade) } \\
F \text { (nrof } x \text { umidade) }\end{array}$ & & & $\begin{array}{l}3,24^{*} \\
1,29^{\text {ns }} \\
0,93^{\text {ns }}\end{array}$ & & \\
\hline $\begin{array}{l}\text { C.V. }(\%) \\
\text { D.M.S. }\end{array}$ & & & $\begin{array}{l}1,79 \\
2,51\end{array}$ & & \\
\hline
\end{tabular}

Médias seguidas de mesma letra maiúscula na coluna e minúscula na linha, não diferem estatisticamente entre si pelo teste Tukey $(\mathrm{P}<0,05)$ * - significativo a 5\% de probabilidade; ** - significativo a 10\% de probabilidade; ns não significativo. DAA: ${ }^{1}$ Dias após a aplicação.

Também aos 42 DAA (Tabela 6), observou excelentes níveis de controle em todos os tratamentos. No entanto, na camada mais superficial $(1 \mathrm{~cm})$, foi observado menores níveis de controle, onde ocorreram chuvas após a aplicação. Este resultado deve estar relacionado, ao fato das espécies do gênero Digitaria apresentarem sementes pequenas e com germinação mais superficial. Carbonari (2007) verificou algumas falhas no controle de Digitaria spp. e Panicum maximum (sementes pequenas) pelo herbicida amicarbazone em época chuvosa, mesmo quando em associação com a palha de cana-de-açúcar. Isto ocorre porque a lixiviação do herbicida amicarbazone germinação pelo herbicida amicarbazone, aos 14 DAA. Botucatu/SP - 2006/07.

\begin{tabular}{|c|c|c|c|c|c|}
\hline \multirow[t]{2}{*}{ Condições de Umidade } & \multicolumn{5}{|c|}{ Profundidade $(\mathrm{cm})$} \\
\hline & 1 & 2 & 3 & 4 & 5 \\
\hline chuva de $15 \mathrm{~mm}$ - aplicação & 99,5 Aa & $97,0 \mathrm{Ab}$ & 99,5 Аa & 98,8 Aab & 99,8 Aa \\
\hline chuva $(15 \mathrm{~mm})$ - aplicação - chuva $(15 \mathrm{~mm}) 7^{1}$ & $94,7 \mathrm{Bb}$ & $98,5 \mathrm{Aa}$ & 99,0 Aa & $98,5 \mathrm{Aa}$ & $100,0 \mathrm{Aa}$ \\
\hline chuva $(15 \mathrm{~mm})$ - aplicação - chuva $(15 \mathrm{~mm}) 14^{1}$ & $98,3 \mathrm{Aa}$ & 99,3 Aa & 99,0 Aa & $98,5 \mathrm{Aa}$ & $100,0 \mathrm{Aa}$ \\
\hline aplicação solo seco - chuva $(15 \mathrm{~mm}) 14^{1}$ & $100,0 \mathrm{Aa}$ & $99,3 \mathrm{Aa}$ & $99,8 \mathrm{Aa}$ & $99,3 \mathrm{Aa}$ & $100,0 \mathrm{Aa}$ \\
\hline $\begin{array}{l}\text { F (profundidade) } \\
\text { F (umidade) }\end{array}$ & & & $\begin{array}{l}3,09^{*} \\
2,88^{*}\end{array}$ & & \\
\hline F (prof. $x$ umidade) & & & $1,95^{*}$ & & \\
\hline $\begin{array}{l}\text { C.V. }(\%) \\
\text { D.M.S. }\end{array}$ & & & $\begin{array}{l}1,64 \\
4,41\end{array}$ & & \\
\hline
\end{tabular}

Médias seguidas de mesma letra maiúscula na coluna e minúscula na linha, não diferem estatisticamente entre si pelo teste Tukey $(\mathrm{P}<0,05) . *$ - significativo a $5 \%$ de probabilidade; $* *$ - significativo a $10 \%$ de probabilidade; ns não significativo. DAA: ${ }^{1}$ Dias após a aplicação. 
Tabela 5. Porcentagem de controle das plantas de Digitaria spp. nas diferentes profundidades de germinação pelo herbicida amicarbazone, aos 28 DAA. Botucatu/SP - 2006/07.

\begin{tabular}{|c|c|c|c|c|c|}
\hline \multirow{2}{*}{ Condições de Umidade } & \multicolumn{5}{|c|}{ Profundidade $(\mathrm{cm})$} \\
\hline & 1 & 2 & 3 & 4 & 5 \\
\hline chuva de 15 mm - aplicação & $98,0 \mathrm{Aa}$ & $99,8 \mathrm{Aa}$ & $100,0 \mathrm{Aa}$ & $100,0 \mathrm{Aa}$ & $100,0 \mathrm{Aa}$ \\
\hline chuva (15 mm) - aplicação - chuva $(15 \mathrm{~mm}) 7^{1}$ & $98,8 \mathrm{Aa}$ & $99,5 \mathrm{Aa}$ & $100,0 \mathrm{Aa}$ & $100,0 \mathrm{Aa}$ & $100,0 \mathrm{Aa}$ \\
\hline chuva (15 mm) - aplicação - chuva $(15 \mathrm{~mm}) 14^{1}$ & $97,3 \mathrm{Aa}$ & $100,0 \mathrm{Aa}$ & $100,0 \mathrm{Aa}$ & $100,0 \mathrm{Aa}$ & $100,0 \mathrm{Aa}$ \\
\hline aplicação solo seco - chuva $(15 \mathrm{~mm}) 14^{1}$ & $96,3 \mathrm{Aa}$ & $100,0 \mathrm{Aa}$ & $100,0 \mathrm{Aa}$ & $100,0 \mathrm{Aa}$ & $100,0 \mathrm{Aa}$ \\
\hline F (profundidade) & & & $1,87^{\mathrm{ns}}$ & & \\
\hline F (umidade) & & & $0,12^{\text {ns }}$ & & \\
\hline F (prof. x umidade) & & & $0,95^{\mathrm{ns}}$ & & \\
\hline C.V. $(\%)$ & & & 2,15 & & \\
\hline D.M.S. & & & 3,02 & & \\
\hline
\end{tabular}

Tabela 6. Porcentagem de controle das plantas de Digitaria spp. nas diferentes profundidades de germinação pelo herbicida amicarbazone aos, 42 DAA. Botucatu/SP - 2006/07.

\begin{tabular}{|c|c|c|c|c|c|}
\hline \multirow[t]{2}{*}{ Condições de Umidade } & \multicolumn{5}{|c|}{ Profundidade $(\mathrm{cm})$} \\
\hline & 1 & 2 & 3 & 4 & 5 \\
\hline chuva de 15 mm - aplicação & $100,0 \mathrm{Aa}$ & $100,0 \mathrm{Aa}$ & $100,0 \mathrm{Aa}$ & $100,0 \mathrm{Aa}$ & $100,0 \mathrm{Aa}$ \\
\hline chuva (15 mm) - aplicação - chuva $(15 \mathrm{~mm}) 7^{1}$ & $100,0 \mathrm{Aa}$ & $100,0 \mathrm{Aa}$ & $100,0 \mathrm{Aa}$ & $100,0 \mathrm{Aa}$ & $100,0 \mathrm{Aa}$ \\
\hline chuva (15 mm) - aplicação - chuva $(15 \mathrm{~mm}) 14^{1}$ & $95,0 \mathrm{Bb}$ & $100,0 \mathrm{Aa}$ & $100,0 \mathrm{Aa}$ & $100,0 \mathrm{Aa}$ & $100,0 \mathrm{Aa}$ \\
\hline aplicação solo seco - chuva $(15 \mathrm{~mm}) 14^{1}$ & $95,0 \mathrm{Ba}$ & $100,0 \mathrm{Aa}$ & $100,0 \mathrm{Aa}$ & $100,0 \mathrm{Aa}$ & $100,0 \mathrm{Aa}$ \\
\hline $\mathrm{F}$ (profundidade) & & & $0,75^{*}$ & & \\
\hline F (umidade) & & & $0,66 *$ & & \\
\hline F (prof. x umidade) & & & $1,08 *$ & & \\
\hline C.V. $(\%)$ & & & 3,18 & & \\
\hline D.M.S. & & & 4,47 & & \\
\hline
\end{tabular}

\section{Conclusões}

Observou-se que para $B$. plantaginea, os melhores resultados de controle foram proporcionados pelos tratamentos com a ocorrência de chuvas, sendo observadas falhas iniciais no controle quando o produto foi aplicado em solo úmido e sem ocorrência de chuvas, nas profundidades de 1,2 e $5 \mathrm{~cm}$, com a recuperação e bons níveis de controle ao final do estudo.

Para a espécie Digitaria spp. foram observados bons níveis de eficácia de controle sobre as plantas nas diferentes condições de aplicação.

\section{Referencias}

CARBONARI, C.A. et al. Avaliação da profundidade de germinação de Brachiaria decumbens em áreas de cana-de-açúcar com aplicação do herbicida Dinamic (amicarbazone). In: CONGRESSO BRASILEIRO DA CIÊNCIA DAS PLANTAS 
DANINHAS, 25, 2006, Brasília. Resumos... Brasília: SBCPD, 2006. p.330.

CARBONARI, C.A. Eficácia do herbicida amicarbazone aplicado em operação conjunta com a colheita de cana-de-açúcar no controle das principais plantas daninhas da cultura. 2007, 120p. Dissertação (Mestrado em Agronomia (amicarbazone). In: CONGRESSO BRASILEIRO DA CIÊNCIA DAS JAVORANI, R.C. et al. Comportamento dos herbicidas atrazina e alachlor aplicados em solo preparado para o cultivo de cana-de-açúcar. Química Nova, v.22, n.1, p.12-21, 1999.

RODRIGUES, B.N., ALMEIDA, F.S. Guia de herbicidas. $5^{\text {a }}$ ed., Londrina, 2005. 592p.

TOLEDO, R.E.B. et al. Dinamic (amicarbazone) - Novo herbicida seletivo para o controle de plantas daninhas em pré e pos emergência na cultura da cana-de-açúcar.

Boletim Informativo - Sociedade Brasileira da Ciência das Plantas Daninhas, v. 10, 2002.

TOLEDO, R.E.B. et al. Performance do herbicida Dinamic (amicarbazone) em área de cana soca infestada com Brachiaria decumbens, Ipomoea grandifolia e Commelina benghalensis. In: CONGRESSO BRASILEIRO DA CIÊNCIA DAS PLANTAS DANINHAS, 24, 2004, São Pedro. Anais... Gramado: SBCPD, 2004, p.156.

VELINI, E.D. Comportamento de herbicidas no solo. In:SIMPÓSIO NACIONAL SOBRE MANEJO DE PLANTA DANINHAS EM HORTALIÇAS, 1992. Botucatu. Resumos... Botucatu, 1992. p. 44-64.
/ Proteção de Plantas) - Faculdade de Ciências Agronômicas. Universidade Estadual Paulista, Botucatu, 2007.

DIAS, T.C.S. et al. Influência da precipitação trimestral acumulada sobre o controle de plantas daninhas pelo herbicida Dinamic PLANTAS DANINHAS, 25, 2006, Brasília. Resumos... Brasília: SBCPD, 2006. p.164. 